\title{
Standard Activities of Wireless Mesh Networks
}

\author{
Longjam Velentina Devi \\ Department of CSE \\ Integral University \\ Lucknow India
}

\author{
Sheeba Praveen \\ Department of CSE \\ Integral University \\ Lucknow India
}

\author{
Prof.Rizwan Beg \\ Department of CSE \\ Integral University \\ Lucknow India
}

\begin{abstract}
Wireless Mesh Networks (WMNs) are gaining increasing popularity due to its features like self organization, selfconfiguration and self-healing. WMNs reduces the authentication time by up to a factor of 3 compared to WLAN, while allowing mobile stations to move without performing additional authentications. Wireless Mesh Networks have been envisioned as an important solution to the next generation wireless networking. As various standards are being specified for different mesh networking technologies in many application areas. Among the various standard organizations, the IEEE standards committee is the most enthusiastic at promoting $\mathrm{WMN}$ as a networking technology in all its aspects. IEEE 802 standards committee includes different working groups for Personal Area Network (PAN), Local Area Network (LAN) and Metropolitan Area Network (MAN) based WMNs. In this paper, we focus on IEEE 802 standards.
\end{abstract}

\section{Keywords- WMN, PAN, LAN, MAN, MAC}

\section{INTRODUCTION}

The IEEE 802 standards committee has defined standards for PANs, LANs and MANs [6]. Many standards are well known and widely used, for example, Ethernet and wireless LAN. Currently, the active IEEE 802 working groups include 802.1, $802.3,802.11,802.15,802.16,802.17,802.18,802.19$, $802.20,802.21$, and 802.22. Except for 802.3 for Ethernet and 802.17 for packet ring, all the other groups are related to wireless networks. More specifically, 802.11, 802.15, 802.16, $802.20,802.21,802.22$ are different groups with a focus on a separate topic related to wireless PAN/LAN/MAN, as explained in Table 1. Since the monitoring of an active participation in, ongoing radio regulatory activities, at both the national and international levels, are so important for the above six working groups for radio based networks, IEEE has a separate technical advisory group, 802.18 , with a focus on radio regulation. In addition, coexistence with current and ongoing standards is critical to the success of any standard, there is a separate technical advisory group, 802.19, dedicated to coexistence. The standards being specified cover various protocol layers such as MAC, routing, roaming, interworking, advanced physical techniques, and so on. Other standards organizations usually either focus on one protocol layer or do not have a special group dedicated to wireless mesh networking technologies.

802.11 based WMNs have been researched for several years now. Driven by the worldwide acceptance of 802.11 wireless LANs and a huge number of 802.11 nodes deployed in various application scenarios, 802.11 based WMNs are probably the most popular ones. The most common solution to wireless mesh networking using 802.11 is to combine layer-3 adhoc routing protocol with 802.11 MAC protocol. Many enhancements have been made to both adhoc routing protocols and the 802.11 MAC protocol so that the performance of WMNs can be as high as possible. However, no standards were specified for 802.11 based wireless mesh network until the establishment of 802.11s standard task group in IEEE

Table 1: IEEE 802 standards working groups for radiobased networks

\begin{tabular}{cl}
\hline \hline $\begin{array}{l}\text { Working } \\
\text { group }\end{array}$ & \multicolumn{1}{c}{ Objective and focus } \\
\hline 802.11 & $\begin{array}{l}\text { This group is for wireless local area networks. } \\
\text { There are more than 24 active task groups for } \\
\text { different topics related to wireless LANs. Among } \\
\text { them, 802.11s is dedicated to developing standard } \\
\text { for meshed wireless LANs. }\end{array}$
\end{tabular}

802.15 This group is for wireless personal area networks. There are more than five active task groups for different topics of wireless PANs. Among them, 802.15.5 is dedicated to standardization of mesh PANs.

802.16 This group is for broadband wireless access in a metropolitan area. Several task groups have completed their projects but more than four tasks groups remain active. Mesh mode has been specified in the completed projects and a new task $802.11 \mathrm{j}$ is dedicated to mobile multihop relay.

802.20 This group specifies physical and MAC layers of an air interface for interoperable mobile broadband wireless access systems, operating in licensed bands below $3.5 \mathrm{GHz}$, optimized for IP-data transport, with peak data rates per user in excess of 1 Mbps. The supported mobility can be up to 250 $\mathrm{km} / \mathrm{h}$ in a MAN environment.

802.21 This is a media independent handoff working group. It develops standards to support handover and interoperability between 802 heterogeneous networks including both 802 and non-802 networks.

802.22 This group targets wireless regional area networks. It is developing a standard for a cognitive radiobased PHY/MAC/air-interface for use by licenseexempt devices on a non-interfering basis in the spectrum that is allocated to the TV broadcast service.

\section{OUTLINES}

This paper describes the various standards for Wireless Mesh Networking in Wireless PAN/LAN/MAN. In Section II we briefly present the wireless mesh networking in LAN. Section 
III describes wireless mesh networking in PAN. Wireless mesh networking in MAN is described in section IV. At the eleventh hour, Section V concludes our paper.

\section{WIRELESS LANS IN MESH NETWORK}

The traditional setup of 802.11 wireless LANs is that the infrastructure of each basic service set (BSS) is connected via Ethernet LANs. Such fixed network architecture limits the flexibility of network deployment and increases cost. Thus, mobility of BSS and multihop networking are needed [9].

Starting from the first IEEE 802.11 standard, the adhoc networking has been specified in the independent basic service set (IBSS) mode. In this mode, stations (STAs) can connect to each other without any central coordinator such as an access point (AP). Moreover, there is no access or connection to the distributed system (DS). Thus, STAs are totally self-contained as an ad hoc network. Such an operating mode has been researched in the field of ad hoc networking. However, it has been realized for a long time, especially in industry, that the IBSS mode is not enough for many interesting application scenarios where ad hoc networking is needed but Internet access and support of client nodes are also necessary. Thus, both infrastructure mode and IBSS mode will be integrated in a new type of multihop network.

To meet the above requirements, wireless mesh networking is needed for 802.11 wireless networks. For years, many companies have developed their proprietary solutions to build up 802.11 based WMNs. Such solutions share several common principles [5].

- The network usually includes three types of nodes: mesh routers, clients, and gateways.

- An ad hoc routing protocol is implemented in mesh routers to work together with 802.11 MAC. Certain radio aware functions may be included in the routing protocol

- The 802.11MAC driver is enhanced in mesh routers to improve multihop performance. Typical examples include fine-tuning CSMA/CA parameters, developing algorithms for multi-radio or directional antennas, etc.

- Certain network configurations are needed to support client access, Internet access, roaming, and so on.

In spite of the similarities, the proprietary solutions are usually not interoperable. In order to resolve such an issue and meet the ever-increasing demands of 802.11 mesh networks, it is critical to develop a standard for 802.11 mesh networks. IEEE 802.11 s serves just this purpose.

Before the establishment of 802.11 s, many companies had started to push a standard for 802.11 mesh networks in 2005 . At the beginning, many proposals were submitted to resolve different issues in 802.11 mesh network. These proposals were finally consolidated and merged into one proposal in early 2006, which provides the basic framework for the current 802.11 task group. Since then, the 802.11 task group has worked on resolving issues in the framework. Draft 1.0 of the $802.11 \mathrm{~s}$ standard has been generated and is currently going through a letter ballot. Because many issues still exist in the $802.11 \mathrm{~s}$ draft, when the official 802.11 standard will be released is still unpredictable.

\subsection{Network Architecture of Wireless LAN:}

In order to understand the network architecture of 802.11 s, we first need to explain 802.11 ESS and its difference from IBSS [1]. An 802.11 ESS consists of multiple BSSs connected through a DS and integrated with wired LANs. The DS service (DSS) is provided by the DS for transporting MAC service data units (MSDU) between APs, between APs and portals, and within the same BSS if MSDU is broadcast/multicast or intended to involve DSS. The portal is the logical point for letting MSDUs from a non-802.11LAN enters the DS. An ESS appears as a single BSS to the logical link control layer at any station associated with one of the BSSs. The 802.11 standard has pointed out the difference between IBSS and ESS. IBSS actually has one BSS and does not contain a portal or an integrated wired LAN since no physical DS is available. Thus, the ESS architecture can meet the needs of client support and Internet access, while IBSS cannot. However, IBSS has the advantage of selfconfiguration and ad hoc multihop networking. Thus, it is a good strategy for developing schemes to combine the advantages of ESS and IBSS. The solution path being taken by IEEE 802.11 s is one such scheme

In 802.11s, a meshed wireless LAN is formed via ESS mesh networking. In other words, BSSs in the DS do not need to be connected by wired LANs. Instead, they are connected via mesh networking, possibly with multiple hops in between. Portals are still needed to interconnect 802.11 wireless LANs and wired LANs. Based on such a concept, the network architecture of $802.11 \mathrm{~s}$ is formed as shown in Figure 10.1. There are three new nodes in this architecture. A mesh point (MP) is an 802.11 entity that can support wireless LAN mesh services. A mesh access point is an MP that can also work as an access point. A mesh portal is a logical point where MSDUs enter and exit the mesh network from and to other parts of the DS such as a traditional 802.11 LAN or from and to a non-802.11 network. Mesh portal includes the functionality of MP. It can be co-located with an 802.11 portal [6]. Because MPs do not have AP functionality but can work as relaying nodes, the meshed wireless LAN is not an ESS anymore. Thus, in a recent 802.11 standard meeting, the suggestion of changing the project authorization request (PAR) of 802.11s was made so that the title of $802.11 \mathrm{~s}$ will be just "Mesh Networking" rather than "ESS Mesh Networking". External Network

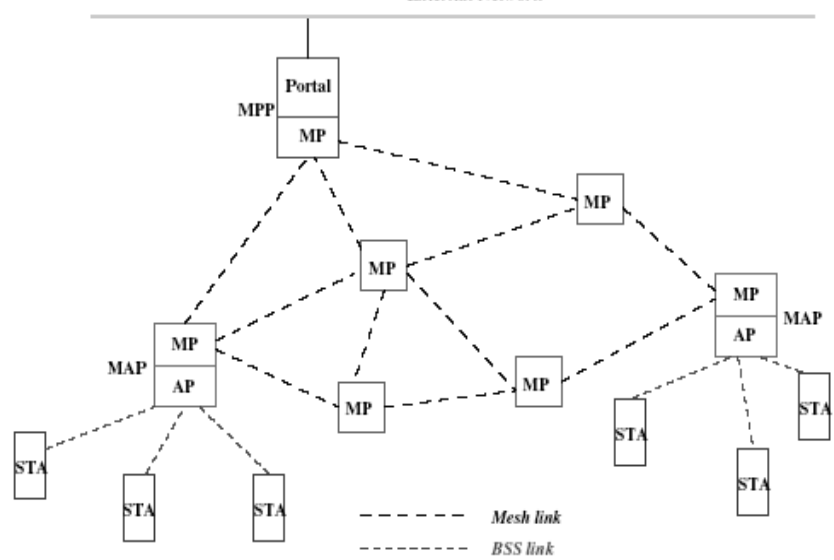

Figure 1: Network architecture of $802.11 \mathrm{~s}$ meshed wireless LANs

The protocol stacks of these three types of nodes are illustrated in Figure 1. The 802.11s MAC is developed based on existing 802.11 MAC for an MP (or the MP module in a Mesh Access Point (MAP) or mesh portal).We can see that the mesh routing protocol of a MP (or the MP module in a MAP or mesh) is located in the MAC layer. In a mesh portal, a layer-3 routing protocol is also needed for path selection from the mesh network to the external network or vice versa. 


\subsection{Framework Overview of Wireless LAN:}

According to the project authorization request (PAR) of 802.11s, the following rules must be followed:

- $\quad$ The standard will be an extension to the IEEE 802.11 MAC.

- The 802.11s defines architecture and protocols to create an 802.11 wireless distribution system (WDS) for ESS mesh networks.

- The ESS mesh is functionally equivalent to ESS connected by wired networks.

- $\quad$ The mesh network can be self-configured.

- The mesh protocol will utilize $802.11 \mathrm{i}$ security and its extension.

- For security, APs in a mesh network are controlled by a single logical administration entity.

- The $802.11 \mathrm{~s}$ amendment will allow the use of one or more radios on each AP in the mesh network.

- The target configuration of $802.11 \mathrm{~s}$ is up to 32 devices participating as mesh points. However, more devices can be supported too.

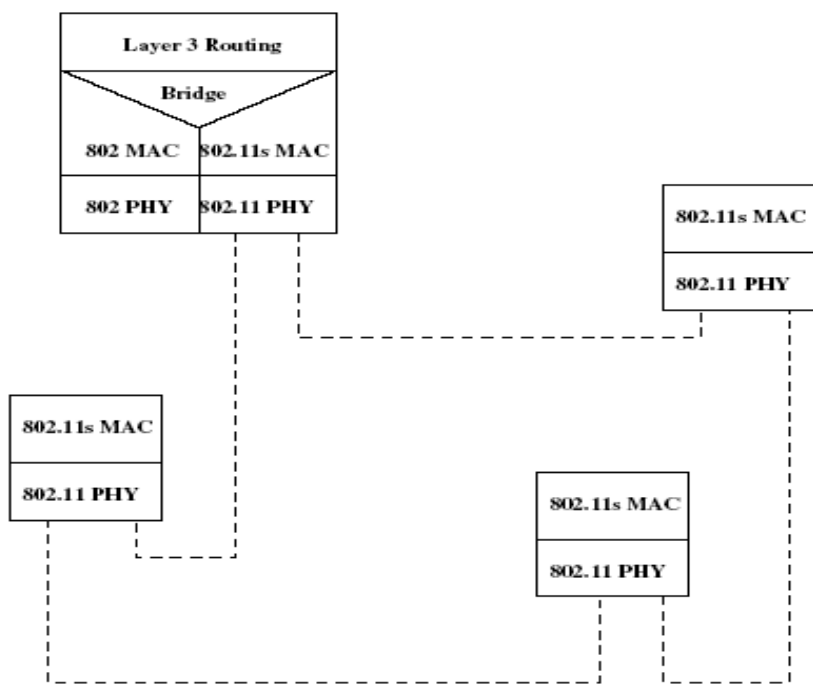

Figure 2: Protocol stack of $802.11 s$

Accordingly, the upcoming 802.11s standard will include the following critical components [2].

- Extension of frame formats for mesh operation: This includes both adding new information elements (IEs) to the existing frames and defining new frames.

- Topology formation of a mesh network: This includes the specifications of how a mesh network is created and how other mesh nodes join or leave the network. Both single channel and multichannel operations will be supported.

- Interworking: Mechanisms for interconnecting the mesh network with other wired networks or wireless networks will be specified.

- MAC: Much functionality need to be specified for the MAC protocol, including MAC enhancement, MAC layer congestion control, power management, multichannel operation, synchronization, and so on.

- Routing in the MAC layer: Since the routing protocol is specified in the MAC layer, MAC address routing is needed. Also, the routing needs to be radio aware. How legacy nodes are supported needs to be specified in the routing framework.

- Security: As always, security is critical for wireless networks.

\section{WIRELESS PANS IN MESH NETWORK}

There are many application scenarios related to wireless PANs. The most well known ones include home networks, office networks, and wireless sensor networks.

Wireless PANs have characteristics such as short distance between nodes and low power consumption. Both MAC and physical layer techniques must take into account such factors. Standard groups such as IEEE 802.15 and other associations such as Bluetooth Special Interest Group (SIG), Wi-Media Forum, UWB Forum, and so on are working on specifications for the protocols in various scenarios for wireless PANs.

Although the IEEE 802.15 standards group is not the only association that works on specifications for wireless PANs, it contains many task groups that cover almost all scenarios for wireless PANs. Also, it is closely related to Bluetooth SIG, Wi-Media Forum, UWB Forum, and so on. Knowing the status in IEEE 802.15 will reveal a big picture of standardization of wireless PANs [3].

Table 2: IEEE 802.15 task groups and standards

\begin{tabular}{llll}
\hline \hline $\begin{array}{l}\text { Standards / } \\
\text { task groups }\end{array}$ & Objective and focus & Status \\
\hline 802.15 .1 & $\begin{array}{l}\text { MAC and physical layer } \\
\text { specifications for wireless }\end{array}$ & Completed \\
& $\begin{array}{l}\text { PANs; corrected and revised } \\
\text { in 2005. }\end{array}$ & \\
Coexistence between 802.15 & Completed \\
wireless PANs and other \\
devices in the same \\
unlicensed band
\end{tabular}

802.15.4a New physical layers for more Active accurate location/range lower power consumptions, and scalable rates

802.15.5 Mesh networks for wireless Active PANs; both low rate and high rate wireless PANs are being considered

\subsection{Network Architecture and Protocol Stack}

In 802.15.5, the meshed wireless PANs can have a topology of full mesh or partial mesh, as shown in Figure 3. There are three types of node in the network: PAN coordinator, 
coordinator, and end device. End devices are connected to their coordinator as a star topology, which is the exactly the same as that in other 802.15 wireless PANs [7]. Coordinators are connected to each other through a mesh topology (either full mesh or partial mesh), which is different from the topology of other 802.15wireless PANs. For example, an 802.15.1 scatternet can also form a multihop wireless PAN, but it is actually a tree-based network topology because of the master/slave mechanism. In 802.15.4, a multihop wireless PAN is formed via the concept of the cluster tree network, which is also a type of tree topology. Neither scatternet nor cluster tree topology has the flexibility of mesh topology, although it is simpler and thus demands lightweight protocols in MAC and routing layers. Lack of such flexibility can result in several other issues such as poor network coverage, low reliability due to no redundant path, high power consumption, etc. Thus, the meshed wireless PANs of 802.15.5 are targeted at:

- Extending network coverage without increasing transmits power or receives sensitivity.

- Enhancing reliability via route redundancy.

- Simplifying network configuration.

- Increasing device battery life with better transmissions and fewer retransmissions.

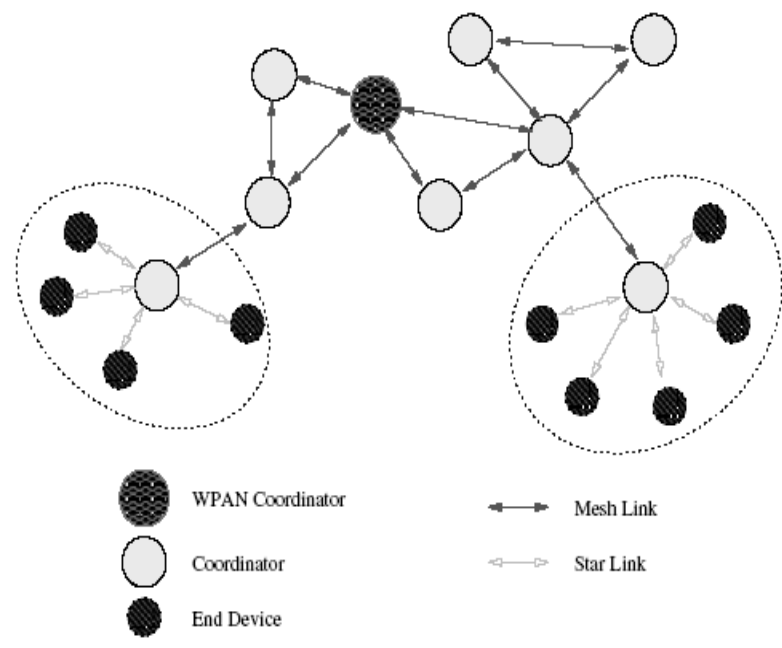

Figure 3: 802.15.5 meshed wireless PANs

Other than the difference in network topology, all other 802.15 wireless PANs have just focused on MAC and physical layers, even for a multihop network. Thus, how to route packets from end to end is not specified. Without a routing protocol this may cause several problems. For example, the routing functionality has to be implemented by vendors, which can cause interoperability issues between products of different vendors. Thus, specifying a routing protocol is one of the most important tasks for the 802.15.5 task group. Thus, in 802.15.5, both MAC and routing protocols need to be specified. The MAC is enhanced based on that of other 802.15 wireless PANs and new routing function is added on top of the enhanced MAC protocol. Since low rate wireless PANs and high rate wireless PANs have different physical layer and MAC layer specifications, the 802.15.5 task group is currently working on separate specifications for them. However, the protocol stack of these two types of mesh network is the same, as shown in Figure 4.

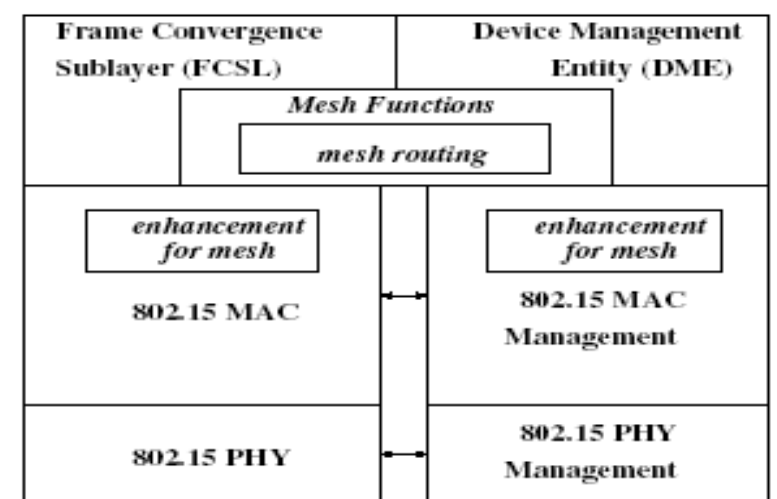

Figure 4: Protocol stack of 802.15.5 meshed wireless PANs

\section{WIRELESS MANS IN MESH NETWORK}

IEEE 802.16 standards are targeted at broadband wireless access in MAN [9]. QoS is always an important concern for any 802.16 standard. Thus, from the beginning, service specific convergence sub-layer, QoS mechanism, perconnection traffic flow management, and scheduling schemes for connections are all covered in the IEEE 802.16 standards [4][5].

Table 3: IEEE 802.16 task groups and standards

\begin{tabular}{ll}
\hline \hline $\begin{array}{l}\text { Standards / } \\
\text { task groups }\end{array}$ & Objective and focus \\
\hline 802.16 .1 & $\begin{array}{l}\text { The first version of the IEEE } \\
802.16 \text { standard. The physical }\end{array}$ \\
& $\begin{array}{l}\text { layer is single carrier and only } \\
\text { PMP mode is considered in the }\end{array}$ \\
& $\begin{array}{l}\text { MAC layer. Frequency bands are } \\
\text { in 10-60 GHz. Only fixed }\end{array}$ \\
& $\begin{array}{l}\text { systems considered } \\
\text { Expand 802.16.1 on system } \\
\text { profiles }\end{array}$
\end{tabular}

$802.16 a$

The first IEEE 802.16 standard in 2-11 GHz. Five options of physical layer is specified. Mesh mode is included in this version

802.16- Provide corrections to IEEE 2004802.16 .1 and consolidate 802.16.1, 802.16c, and 802.16a. Only fixed systems are considered. This work was done in task group 802.16d and 802.16-REVd

802.16f Expand 802.16-2004 on MIB

Approved in September 2005

802.16e Expand 802.16-2004 to allow for mobility in subscriber stations

Approved in December 2002

Approved in January 2003

Approved in June 2004

Approved in December 2005

802.16g Part of management task group. Amend 802.16 on management plane procedures and services

Part of management task group. Active Amend 802.16 on mobile MIB

802.16k Part of management task group. Amend 802.1D for 802.16

Active

Active 
$802.16 \mathrm{~h}$ bridging functionality

$802.16 \mathrm{j}$
Specify coexistence procedures Active in license-exempt bands

Enhance 802.16/2004 and 802.16e to support mobile multihop relay. Enhancements are carried out in wireless MANOFDMA physical layer and MAC layer. Relay stations and base stations need to be interoperable. No change is needed for subscriber stations

\subsection{Network Architecture of Mesh Mode}

In PMP mode, direct communications links exist only between a base station (BS) and subscriber stations (SSs) [8] [10]. However, in mesh mode direct communications among SSs, and between a BS and SSs, are all possible. Thus, BSs and SSs become mesh BSs and mesh SSs in mesh mode. A mesh BS is the BS that has connection to backhaul services. Thus, a generic architecture for mesh mode is as follows. A top tier 802.16 BS node covers a few mesh BSs via the PMP mode, and in the second tier a mesh BS provides backhaul access for many mesh SSs. The mesh BS and its mesh SSs form a cluster of mesh nodes. Within the cluster, mesh networking is used to connect the different nodes. Direct communications between mesh nodes in different clusters are not necessary. When a new node joins the network, the network entry process will help the new node find the best mesh BS of a particular cluster. Thus, to investigate the mesh mode of 802.16 , we can focus on one mesh cluster that consists of one mesh BS and a number of mesh SSs.

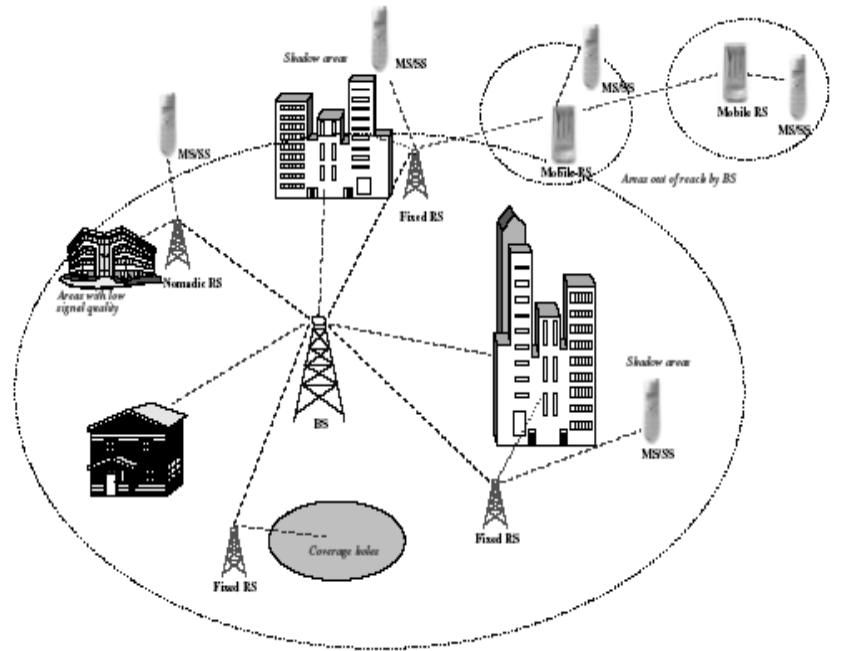

Figure 6: The network architecture of 802.16 mobile multihop relay

\section{CONCLUSION}

As we focused on the standard activities within the IEEE 802 standards committee, specifications related to WMNs but produced by industry alliances or forums are also presented. Most IEEE 802 standards are focused on MAC and physical layer specifications; issues above these two layers are specified in 802.1. Currently 802 LAN / MAN architecture, internetworking among 802 LANs, MANs and other wide area networks, 802 Security, and 802 overall network management and so on are all specified in 802.1. As far as WMNs are concerned, only IEEE $802.11,802.15,802.16$ are actually developing standards for this purpose. As till date no standard on WMNs is really available for implementation, finalizing standards is still an ongoing effort. However, drafts of these standards have been released in different working groups and their task groups. We present the latest work on these drafts. However, the process of standardizing WMNs is so complicated that many issues in wireless mesh networking have not yet been resolved.

\section{ACKNOWLEDGEMENT}

The Authors would like to thank Mr.Sahil Seth PEC (Cyber Security) for his guidance, also Mr Dhruba Shankar Ray for giving support.

\section{REFERENCES}

[1] Liu Pu, "Design of Wireless Communication Protocol for Home LAN" in proceeding of 2009 International Symposium on Intelligent Ubiquitous Computing and Education, May 2009,pp. 374-377.

[2] Hyun Lee, Je-Hoon Lee, Seok-Man Kim , Kyoung-Rok Cho, "Implementation of IEEE 802.11a Wireless LAN" in proceeding of 2008 Third International Conference on Convergence and Hybrid Information Technology, November 2008,pp. 291-296.

[3] SeongHan Shin , Hanane Fathi , Kazukuni Kobara, Hideki Imai, "A Secure Group Communication Framework in Private Personal Area Networks (PPANs)" in proceeding of Third International Conference on Wireless and Mobile Communications (ICWMC'07), March 2007, pp. 59.

[4] Thierry Peyre, Rachid ElAzouzi, "Performance Analysis of Single Cell IEEE 802.16e Wireless MAN" in proceeding of 32nd IEEE Conference on Local Computer Networks(LCN2007), October 2007, pp. 262263.

[5] IEEE 802.11s Task Group 2006b Draft amendment to standard for information technolog telecommunications and information exchange between systems - LAN/MAN specific requirements - Part 11: wireless medium access control (MAC) and physical layer (PHY) specifications: Amendment: ESS mesh networking. IEEE P802.11s/D1.00-2006.

[6] I. F. Akyildiz, X. Wang, and W. Wang, "Wireless mesh networks: a survey," Computer Networks and ISDN Systems, vol. 47, no. 4, pp. 445-487, Mar. 2005.

[7] Miklos Aurel Ronai , Kristof Fodor, Gergely Biczok, Zoltan Turanyi, Andras Valko, "MAIPAN - Middleware for Application Interconnection in Personal Area Networks" in proceeding of The Second Annual International Conference on Mobile and Ubiquitous Systems: Networking and Services, July 2005, pp. 494498.

[8] Greg Goth, "Wireless MAN Standard Signals Next-Gen Opportunities" in proceeding of IEEE Distributed Systems Online, August 2004, pp. 4.

[9] LAN-MAN Standards Committee of the IEEE Computer Society, Wireless Medium Access Control (MAC) and Physical Layer (PHY) Specifications for Low-Rate Wireless Personal Area Networks (LR-WPANs), IEEE, 2003.

[10] IEEE 802.16-2001, "IEEE Standard for Local and Metropolitan Area Networks, Part 16: Air Interface for Fixed Broadband Wireless Access Systems," Apr. 8, 2002. 